\title{
PRF as a pulpotomy medicament in a permanent molar with pulpitis: a case report
}

\author{
Dr.Seema Pathak ${ }^{1}$ Dr.Pradnya Bansode ${ }^{2}$, Dr.Chetan Ahire ${ }^{3}$ \\ 1(Associate professor, Department of Conservative Dentistry and Endodontics Govt. Dental College and \\ Hospital, Aurangabad (M.S), College, MUHS, India) \\ 2, ( Professor and Head, Department of Conservative Dentistry and Endodontics, Govt. Dental College and \\ Hospital, Aurangabad(M.S), College, MUHS, India) \\ 3(Pg student, Department of conservative dentistry and endodontics, Govt. Dental College and Hospital, \\ Aurangabad(M.S), College, MUHS, India)
}

\begin{abstract}
Aim: To discuss the clinical and radiographic success of a pulpotomy with PRF, in a human immature permanent molar tooth.

Summary: A 14-year-old male patient reported to the Department of Conservative Dentistry and Endodontics with established pulpitis in tooth no. 47. The tooth had a carious pulp exposure, with a history of lingering pain. After isolation, caries removal and pulp exposure, pulpotomy with PRF was performed and a permanent restoration was placed immediately. At the first recall $(+1$ day), no postoperative pain was reported. At 6 months recall, the tooth responded positively to pulp sensibility tests, and radiographic examination revealed a normal periodontal ligament space.
\end{abstract}

Keywords: growth factors, immature root apex, platelet-rich fibrin, pulpitis, pulpotomy.

\section{Introduction}

The vitality of the dentine-pulp complex is fundamental to the health of tooth and is a priority for targeting clinical management strategies. Preserving the pulp is important in the treatment of carious exposures in young permanent teeth or in the complex root canal systems of primary molars[1]. Exposures may result from caries, iatrogenic mishaps or traumatic injuries [2].

Several case series have suggested pulpotomy as a viable treatment for pulp exposures with pulpitis; the rationale being the healing potential of the remaining radicular tissue and the biocompatibility of pulpotomy agents[3] . Therefore, it is important to develop biocompatible treatments directed at maintaining pulp vitality and increasing tooth longevity. To increase the success rate, a critical need exists to develop new biologically based therapeutics that reduce pulp inflammation and promote the formation of dentine pulp tissues.

Platelet-rich fibrin (PRF) was first described by Choukroun et al. (2006)[4]. It has been referred to as a secondgeneration platelet concentrate, which has been shown to have several advantages over traditionally prepared platelet-rich plasma. Its chief advantages include ease of preparation and no biochemical handling of blood, which makes this preparation strictly autologous .

This case report presents the use of PRF as a pulpotomy material in treating pulpitis in a human permanent molar.

\section{Case report}

A 14-year-old male patient reported to the Department of Conservative Dentistry and Endodontics, GDC\&H Aurangabad with pain in the lower right posterior region of jaw. Pain was spontaneous, intense \& lingering to cold stimulus with no history of swelling \& night pain . On clinical examination deep occlusal caries were seen on right mandibular second molar(47). Tooth was mildly tender to percussion. An IOPA x-ray revealed deep occlusal caries invading the pulp with immature roots $\&$ thin root dentinal walls.

The diagnosis of pulpitis was determined on the basis of clinical and radiographical assessment.The patient was informed about the treatment modality i.e. coronal pulpotomy using PRF. After obtaining written consent from the patient, PRF was prepared by drawing the required amount of blood into a $10-\mathrm{mL}$ test tube without an anticoagulant and centrifuged immediately using a table top centrifuge (Dept. of Oral Pathology \& Microbiology GDC\&H Aurangabad) for $12 \mathrm{~min}$ at $571.54 \mathrm{~g}$. The resultant product consisted of the following three layers: (Fig. 2)

- Acellular platelet poor plasma at the top of the tube;

- Fibrin clot (PRF) in the middle of the tube; and

- Red blood corpuscles at the bottom of the tube. 
Because of the absence of an anticoagulant, blood begins to coagulate as soon as it comes in contact with the glass surface. Therefore, for successful preparation of PRF, rapid blood collection and immediate centrifugation, before the clotting cascade is initiated, are absolutely essential. PRF was obtained in the form of a membrane by squeezing out the fluids in the fibrin clot.

Tooth 47 was first anaesthetized with Lidocaine 2\% and adrenaline 1/80 000 (Astra- Zeneca Pharma India Ltd, Bangalore, India) and isolated with a rubber dam. Pulpotomy was performed with a round bur in a high-speed handpiece with copious irrigation; coronal pulp tissue was removed to the level of pulp chamber floor. Haemostasis was achieved by irrigating the cavity with sterile saline and cotton pellets (Fig. 3). The blood clotfree pulpal wound was covered with a small piece of PRF (Fig.4). An approximately $2 \mathrm{~mm}$ thick layer of MTA (ProRoot; Dentsply Tulsa Dental Specialty, Tulsa, OK, USA) was placed over the PRF (Fig. 5) and a final restoration of glass-ionomer cement was placed. An immediate postoperative radiograph was obtained. The patient was recalled next day for evaluation of postoperative pain if any. The patient had no pain or discomfort. And at 6 months recall, the tooth responded positively to pulp tests, and radiographic examination revealed normal periodontal ligament space and trabecular bone pattern approaching normal. Tooth might be totally asymptomatic as patient has not reported for further follow up.

\section{Discussion}

Pulpotomy is a universally accepted treatment for teeth with incompletely formed roots involving pulpal exposure [5,6]. In recent years, MTA has been introduced for pulpotomy in primary molars [10]. A number of laboratory studies have been conducted to evaluate the biocompatibility of MTA by measuring various parameters such as proliferation and viability using different types of cells in direct and/or indirect contact with MTA. It has demonstrated good biocompatibility [8], excellent sealing ability [7], and stimulation of healing in the pulpal tissue (Asgary et al. 2008). But MTA in its freshly mixed state shows a higher cytotoxicity[11,12] which could be due to its high $\mathrm{pH}$ [13]. Therefore, it is important to develop a new biocompatible material directed at maintaining a pulp vitality and increasing tooth longevity (Wang et al. 2010)[14]. One such biologically based therapeutic is PRF. PRF is a second-generation platelet concentrate widely used to accelerate soft and hard-tissue healing. Its advantages over the better known platelet-rich plasma (PRP) include ease of preparation/application, minimal expense and no biochemical modification (no bovine thrombin or anticoagulant is required).

Platelet-rich fibrin is a strictly autologous fibrin matrix containing a large quantity of platelet and leucocyte cytokines (Sunitha Raja \& Munirathnam Naidu 2008)[15]. Growth factors play a pivotal role in signalling the events of tissue formation and repair in the dentine-pulp complex. They are responsible for signalling many of the key events in tooth morphogenesis and differentiation, and recapitulation of these processes allows tissue regeneration (Smith 2003)[16].

In the current case, an effort was made to use such growth factors to help in repair of a tooth with pulpitis. As discussed earlier, PRF was prepared with the patients' own blood and was placed in the pulp chamber after a pulpotomy procedure. A layer of MTA was placed over PRF and the final restoration of glassionomer cement was placed immediately. MTA was chosen as it is hydrophilic and requires moisture to set, which is a favourable property when there is potential for moisture contamination in the clinical setting (Gancedo-Caravia \& Garcia-Barbero 2006)[17].

The potential theory behind the success of the presented case could be attributed to a study conducted by Wang et al. (2010) that the pulp cells residing in pulp clinically diagnosed with pulpitis might still have stem cell potential similar to healthy pulp cells and therefore might be a resource for autologous pulp regeneration. These findings suggest exciting opportunities for biologically based therapeutic approaches to dental tissue repair as well as providing valuable insights into how natural regenerative processes may be operating in the tooth. Further research on this topic is required with regard to the histological assessment of such treated teeth on a larger sample size.

IV. Figures

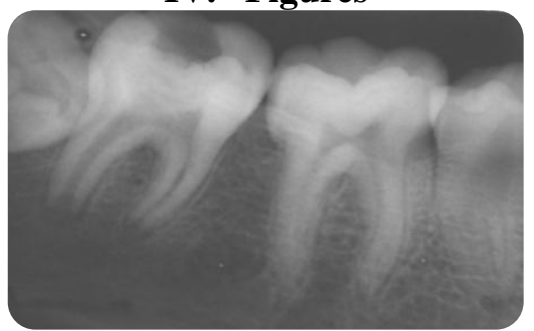

Figure: 1 IOPA x-ray showing deep occlusal caries invading the pulp with immature roots \& thin root dentinal walls 

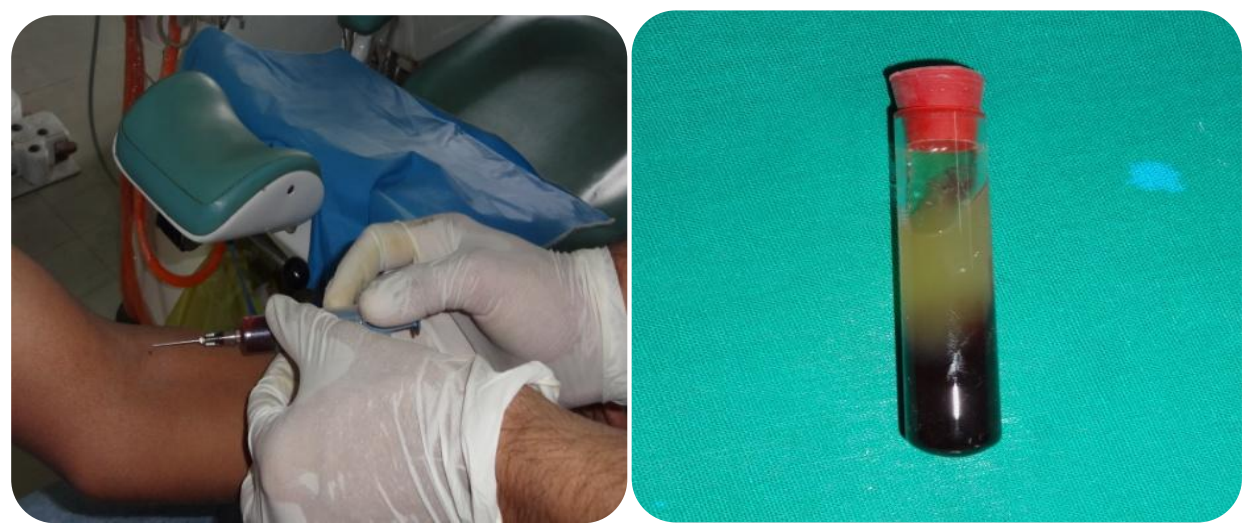

Figure 2: Venous blood collection with 22 gauge sterile niddle syringe \& preparation of PRF

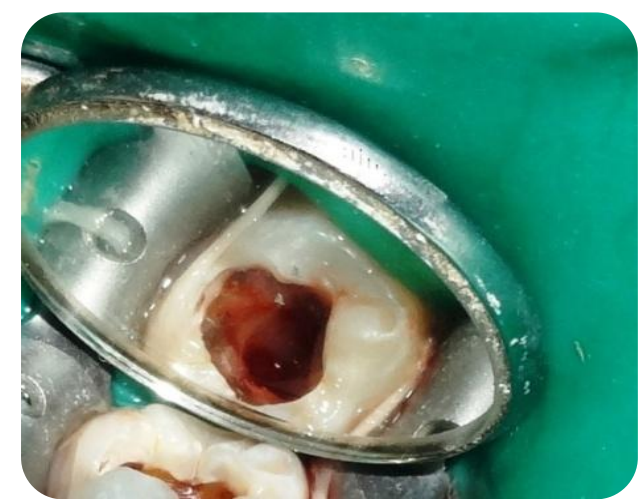

Figure 3: After removal of coronal pulp with tooth no.47

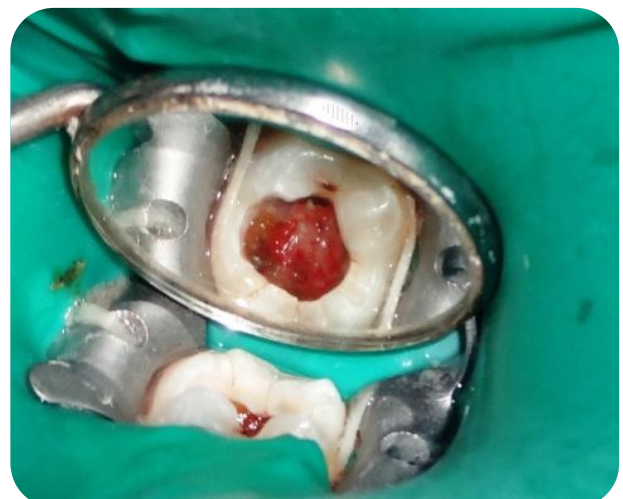

Figure 4: After placement of PRF in coronal pulp chamber

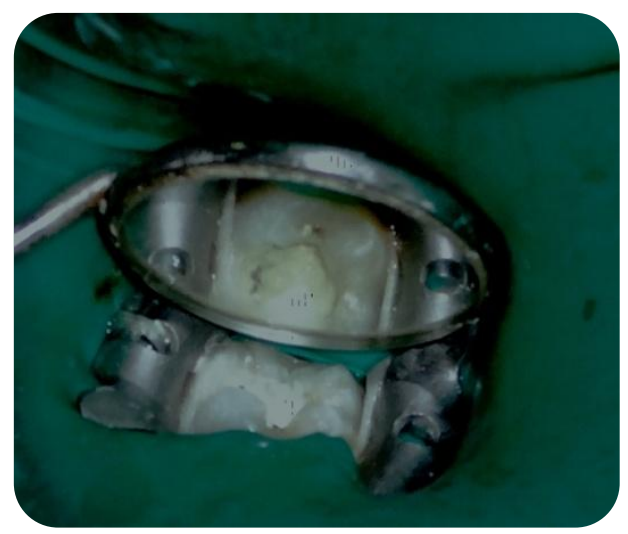

Figure 5: After placement of MTA over PRF 


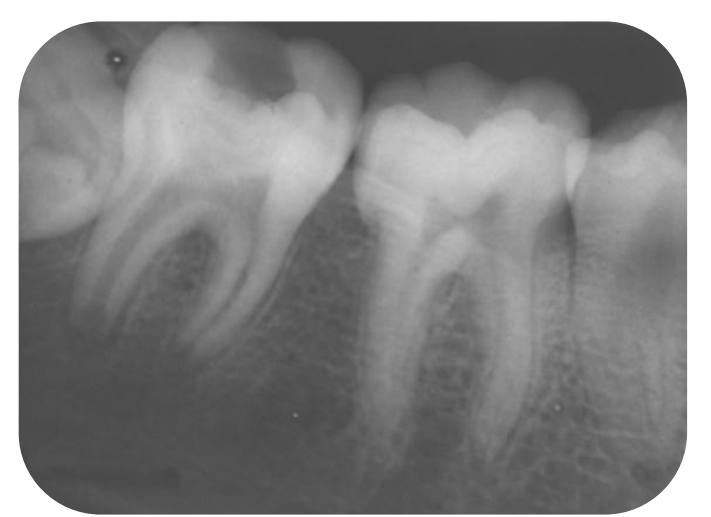

Figure 6: IOPA x-ray immediately after placement of MTA

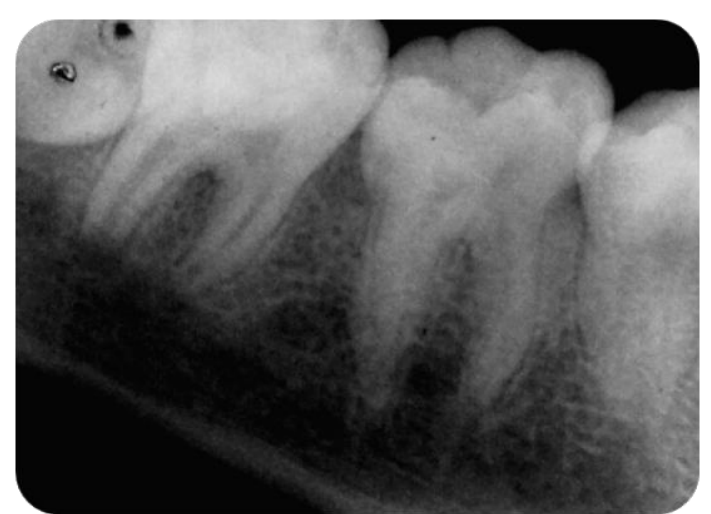

Figure 7: IOPA x-ray after final restoration

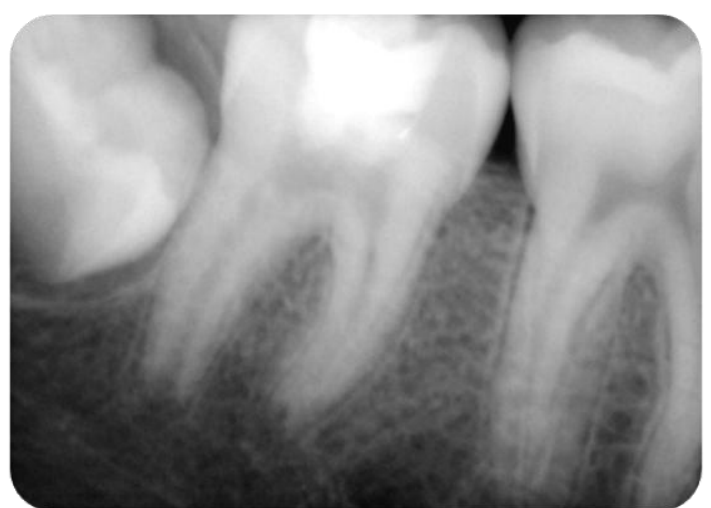

Figure 8: IOPA x-ray showing continued root formation, follow up after 6 months.

\section{Conclusion}

The slow polymerizing potential of PRF and the fibrin technology accounts for a favourable physiologic structure to support healing. Growth factors can help in providing a blue print for tissue regeneration within tooth, thus helps in dental tissue repair. It can be concluded that there is a reasonable biological argument to carry out pulpotomy as a possible alternative treatment in mature permanent teeth with pulpitis. Further studies (histological and clinical) can add significant weight to this argument. 


\section{References}

[1]. Witherspoon DE, Small JC, Harris GZ (2006) Mineral trioxide aggregate pulpotomies: a case series outcomes assessment. Journal of American Dental Association 137, 610-8.

[2]. Bakland LK (2002) Endodontic considerations in dental trauma. In: Ingle JI, Bakland LK, eds. Endodontics. Toronto: BC Decker Inc, pp. 795-844.

[3]. Eghbal MJ, Asgary S, Ali Baglue R, Parirokh M, Ghoddusi J (2009) MTA pulpotomy of human permanent molars with irreversible pulpitis. Australian Endodontic Journal 35, 147-52.

[4]. Choukroun J, Diss A, Simonpieri A, et al. (2006) Platelet-rich fibrin (PRF): a second-generation platelet concentrate. Part IV: clinical effects on tissue healing. Oral Surgery Oral Medicine Oral Pathology Oral Radiology and Endodontics 101, E56-60.

[5]. Camp JH, Fuks AB (2006) Pediatric endodontics. In: Cohen S, Hargreaves KM, eds. Pathway of the pulp, 9th edn. St. Louis: CV Mosby, p. 838 .

[6]. Amir FA, Gutmann JL, Witherspoon DE (2001) Calcific metamorphosis: a challenge in endodontic diagnosis and treatment. Quintessence International 32, 447-55.

[7.] Aqrawabi J (2000) Sealing ability of amalgam, super EBA cement, and MTA when used as retrograde filling materials. British Dental Journal 188, 266-8.

[8]. Asgary S, Eghbal MJ (2010) A clinical trial of pulpotomy vs. root canal therapy of mature molars. Journal of Dental Research 89, 1080-5.

[9] Asgary S, Eghbal MJ, Parirokh M, Ghanavati F, Rahimi H (2008) A comparative study of histologic response to different pulp capping materials and a novel endodontic cement. Oral Surgery Oral Medicine Oral Pathology Oral Radiology and Endodontics $106,609-14$

[10] Messer LB (2008) Mineral trioxide aggregate as a pulpotomy medicament: an evidence-based assessment. European Archives of Paediatric Dentistry 9, 58-73.

[11]. Haglund RJ, He J, Jarvis J, Safavi KE, Spangberg LSW, Zhu Q (2003) Effects of root-end filling materials on fibroblasts and macrophages in vitro. Oral Surgery, Oral Medicine, Oral Pathology, Oral Radiology, and Endodontics 95, 739-45.

[12]. Balto HA (2004) Attachment and morphological behaviour of human periodontal ligament fibroblasts to mineral trioxide aggregate: a scanning electron microscope study. Journal of Endodontics 30, 25-9.

[13]. Camilleri J (2008) Characterization of hydration products of mineral trioxide aggregate. International Endodontic Journal 41, 40817.

[14]. Wang $\mathrm{Z}$ et al. (2010) Putative stem cells in human dental pulp with irreversible pulpitis: an exploratory study. Journal of Endodontics 36, 820-5.

[15]. Sunitha Raja V, Munirathnam Naidu E (2008) Platelet-rich fibrin: evolution of a second-generation platelet concentrate. Indian Journal of Dental Research 19, 42-6.

[16] . Smith AJ (2003) Vitality of the dentin-pulp complex in health and disease: growth factors as key mediators. Journal of Dental Education 67, 678-89.

[17]. Gancedo-Caravia L, Garcia-Barbero E (2006) Influence of humidity and setting time on the push-out strength of mineral trioxide aggregate obturations. Journal of Endodontics 32, 894-6. 